\title{
Laparoscopic perigastric mesogastrium excision technique for radical total gastrectomy
}

\author{
Chang-Yue Zheng ${ }^{1,2}$, Zhi-Yong Dong ${ }^{3}$, Xian-Tu Qiü ${ }^{1,2}$, Long-Zhi Zheng ${ }^{1,2}$, Jian-Xin Chen ${ }^{1,2}$, Bin Zu ${ }^{1,2}$, Wei Lin ${ }^{1,2}$ \\ ${ }^{1}$ Department of Gastrointestinal Surgery, the Affiliated Hospital of Putian University, Putian, Fujian Province, China \\ ${ }^{2}$ Gastrointestinal Surgery Research Institute, the Affiliated Hospital of Putian University, Putian, Fujian Province, China \\ ${ }^{3}$ Department of Gastrointestinal Surgery, the First Affiliated Hospital of Jinan University, Guangzhou, China
}

Videosurgery Miniinv 2019; 14 (2): 229-236

DOI: https://doi.org/10.5114/wiitm.2018.77874

\begin{abstract}
Introduction: Complete mesogastrium excision (CME) of the perigastric mesogastrium and dissection of lymph nodes (LNS) no. 10 and no. 11 remain technically challenging aspects of laparoscopic radical total gastrectomy (LRTG) plus CME. To address some of these difficulties, we introduced the laparoscopic perigastric mesogastrium excision technique (LPMET) and the concept of the "enjoyable space" to partly modify the procedures of conventional radical surgery and characterize the perigastric space and the surgical plane as well as its boundaries.

Aim: To introduce the laparoscopic perigastric mesogastrium excision technique (LPMET) and the "enjoyable space" when undergoing laparoscopic radical total gastrectomy.

Material and methods: From July 2016 to June 2017, 79 cases of upper gastric cancer that were treated by laparoscopic D2 gastrectomy plus CME were investigated. The retrospective database included the patient characteristics, intraoperative and postoperative outcomes, and morbidity and mortality rates depending on the completeness of their medical records.

Results: Laparoscopic D2 gastrectomy plus CME was successfully performed in all 79 cases. The mean surgical time was $232.5 \pm 46.0 \mathrm{~min}$, and the intraoperative blood loss was $67.6 \pm 52.3 \mathrm{ml}$. A total of $2245 \mathrm{LNs}$ were retrieved (mean $28.1 \pm 10.8$ retrieved from each specimen). The mean postoperative hospital stay was $10.3 \pm 1.6$ days. The postoperative morbidity rate was $17.7 \%$. After a median follow-up period of 12 months, one patient experienced liver metastasis; of the other 78 patients, none died or experienced tumor recurrence or metastasis.

Conclusions: Laparoscopic perigastric mesogastrium excision technique and the "enjoyable space" could be a novel, minimally invasive approach and space to achieve CME and provide benefit for the dissection of LNs no. 10 and no. 11 .
\end{abstract}

Key words: gastric cancer, laparoscopic surgery, D2 lymphadenectomy, enjoyable space.

\section{Introduction}

With the rapid development of minimally invasive surgery, laparoscopic gastrectomy with D2 lymphadenectomy, including dissection of the no. 10 and no. 11 lymph nodes (LNs), has become more widely accepted and confirmed as safe and feasible surgery for patients with advanced upper gastric cancer
(AUGC) [1-4]. Although Huang's three-step maneuver demonstrated the concrete operation steps and the skill of laparoscopic spleen-preserving splenic hilar lymphadenectomy [5], there is still a blind spot within the precut area before LNs no. 10 and no. 11 are dissected along only the splenic vessel trunk and branches, which may lead to injury of the splenic vessels or peripheral organs. Moreover, some stud-

Address for correspondence

Wei Lin MD, PhD, Department of Gastrointestinal Surgery and Gastrointestinal Surgery Research Institute, the Affiliated Hospital

of Putian University, 999 Dongzhen Road, Putian 351100, Fujian, China, phone: +86 0594 2730373, fax: +86 0594 2293910,

e-mail: linwbj@outlook.com 
ies have indicated that the appearance of cancer cells in the mesogastrium with perigastric adipose tissue (named "metastasis V"), which is considered a risk factor to long-term survival, can be detected in both patients with advanced gastric cancer (24\%) and those with early gastric cancer $(2.5 \%)[6,7]$. Thus, complete mesogastrium excision (CME) is particularly important for radical total gastrectomy. Although the "table model" has been proposed to describe the relationship between the stomach and gastric mesenteries [8], the standardized surgical plane and its detailed procedure for CME are still unclear. Therefore, it is necessary to explore a new approach that may complete the dissection of LNs no. 10 and no. 11 and provide CME access. In the experience of our center, we discovered a latent space between the left renal fascia (also called Gerota's fascia) and the posterior aspect of the lesser peritoneal sac; it is a natural avascular zone containing loose connective tissue that we called the "enjoyable space".

\section{Aim}

This study aimed to introduce the laparoscopic perigastric mesogastrium excision technique (LPMET) and the "enjoyable space" and share the experience of 79 AUGC patients (preoperative assessment) undergoing laparoscopic radical total gastrectomy (LRTG) plus CME.

\section{Material and methods}

\section{Patients}

There were 79 patients with upper gastric cancer (T1-T4a, NO-N3, MO, AJCC TNM staging [9]) who underwent the LPMET surgery in the gastrointestinal surgery department of the affiliated hospital of Putian University between July 2016 and June 2017. Upper gastric cancer was diagnosed by endoscopic biopsy specimen analysis. Preoperative imaging studies were routinely performed following endoscopic examination, computed tomography (CT) scanning and abdominal ultrasonography (US). Endoscopic examination and CT scanning were performed to assess the depth of tumor invasion and local LN enlargement. Patients with distant metastasis or preoperative enlargement and LN integration were excluded. None of the patients received prior abdominal surgery or preoperative chemoradiation therapy. This study was performed under a protocol approved by the Ethics Committee at the Affiliated Hospital of Putian University (approval number: 20080323) and was performed after explaining the study advantages and risks to the patients and obtaining informed consent. Surgical operations were performed by Dr. Wei Lin, who has surgical experience of more than 300 cases in laparoscopy-assisted D2 gastrectomy. Blood loss during surgery was measured by estimating the volume of blood in the suction container and weighing blood-soaked gauze. The tumor location was confirmed again, and the maximum diameter was measured immediately after surgery.

\section{Surgical technique}

\section{Position and trocar placements}

Patients were placed in the reverse Trendelenburg position with their heads elevated approximately 15 to $20^{\circ}$. The surgeon stood on the patient's left side, with the assistant on the patient's right side and the camera operator between the patient's legs. A 10-mm trocar was inserted $1 \mathrm{~cm}$ below the umbilicus for the laparoscope, a $12-\mathrm{mm}$ trocar was inserted into the left preaxillary line $2 \mathrm{~cm}$ below the costal margin as a major hand port, and three $5-\mathrm{mm}$ trocars were inserted into the right preaxillary line $2 \mathrm{~cm}$ below the costal margin, the left midclavicular line $2 \mathrm{~cm}$ above the umbilicus and its contralateral site for assistance and exposure (Figure 1).

\section{The procedures}

1. The gastrocolic ligament was routinely divided using an ultrasonic scalpel along the border of the transverse colon toward the left to expose the tail of the pancreas, and toward the right in a similar manner to separate the gastrocolic fusional fascia and find the gastroduodenal artery (GDA). Then, the right gastroepiploic vein, right gastroepiploic artery and right gastric artery (LN no. 5) were vascularized and divided. Fatty lymphatic tissue enveloping or in contact with the pylorus, common hepatic artery and the proper hepatic artery was removed en bloc to dissect LNs no. 6 , no. 8 a and no. 12 a.

2. Separating the "enjoyable space":

Step 1. The entrance:

Exposure: The assistant persistently clamped and pulled the left gastric artery (LGA) upward to main- 
tain proper tension, and placed the greater omentum behind the stomach to keep the visual field clear.

Operation: The surgeon gently pressed the body of the pancreas and peeled the serosa covering the pancreas toward the superior border of the pancreas to expose the root of the LGA and the splenic artery, and reveal the entrance, defined by the LGA on the right side and the splenic artery on the lower side (Photo 1 A). Next, the surgeon cut the loose connective tissue within it to gain access to the "enjoyable space" and find the left Gerota's fascia.

Step 2. The lower border:

Exposure: The assistant used stomach grasping forceps to insert into the entrance and push the posterior gastric wall upward, while the surgeon gently pressed the pancreas and the spleen artery or pushed the surrounding tissue lower to expose the left Gerota's fascia and the splenic vessels.

Operation: The surgeon meticulously cut the connective tissues along the surface of the left Gerota's fascia to expand the "enjoyable space" and expose the profile of the splenic artery. Then, the surgeon gently dissected the membrane-like tissue along the surface of the splenic artery toward the tail of the pancreas. The splenic artery was defined as the lower border (Photo $1 \mathrm{~B}$ ).

Step 3. The right border:

Exposure: The assistant continuously pushed the posterior gastric wall upward. The surgeon gently pressed the LGA rightward to reveal the right crus of the diaphragm.

Operation: The surgeon denuded the right crus of the diaphragm, which was defined as the right border, from the back of the LGA to the right side of the esophageal hiatus (Photo $1 \mathrm{C}$ ).

Step 4. The upper border:

Exposure: The assistant pushed the posterior wall of the body and fundus of the stomach upward and flexibly adjusted the sustain point and the stress direction to maintain proper tension. The surgeon's left hand pushed and tensed the crura of the diaphragm and the left Gerota's fascia to reveal the upper perigastric space.

Operation: The surgeon meticulously dissected the posterior gastric mesentery from the surface of the crura of the diaphragm to expose the gastrophrenic ligament (GPL). Subsequently, the posterior esophageal wall was exposed by separating it deep into the esophageal hiatus. The connective line on the esophageal hiatus, the surface of the left crus of

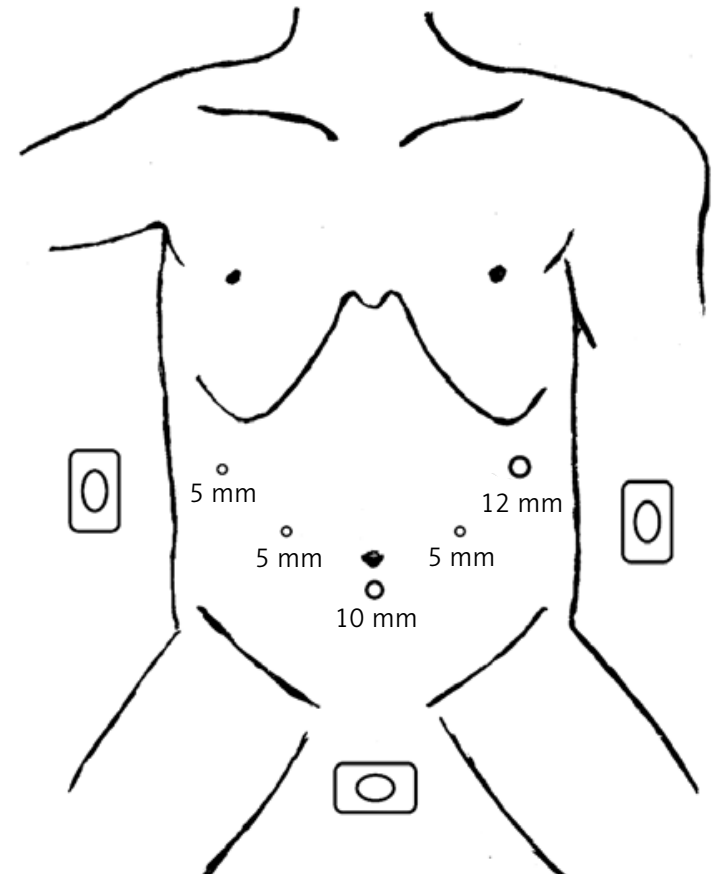

Figure 1. Positions of operators and trocar placements for laparoscopic total gastrectomy. The surgeon stands on the patient's left side, the assistant stands on the patient's right side, and the camera operator stands between the patient's legs

the diaphragm and the GPL emerged as the upper border (Photo $1 \mathrm{D})$.

Step 5. The left border:

Exposure: The assistant continuously pushed the posterior gastric wall upward. The surgeon's left hand pushed the left posterior gastric wall toward the upper left to fully reveal the left perigastric space and maintain proper tension.

Operation: The surgeon continuously extended the surgical plane along the surface of the left Gerota's fascia toward the left to fully expose the posterior edge of the middle-upper spleen, which was defined as the left border (Photo 1 E). At this point, the procedure for separating the "enjoyable space" was completed.

3. The celiac trunk and the LGA were completely skeletonized to dissect LNs no. 7 and no. 9, and the LGA was divided at its root. The overall view of the "enjoyable space" was visible (Photo $1 \mathrm{~F}$ ).

4. The stomach was lifted toward the head to expose the gastropancreatic fold. The fatty lymphatic tissue around the splenic vessels was removed with the non-functional face of the ultrasonic scalpel along its surface to dissect LN no. 11. Meanwhile, 

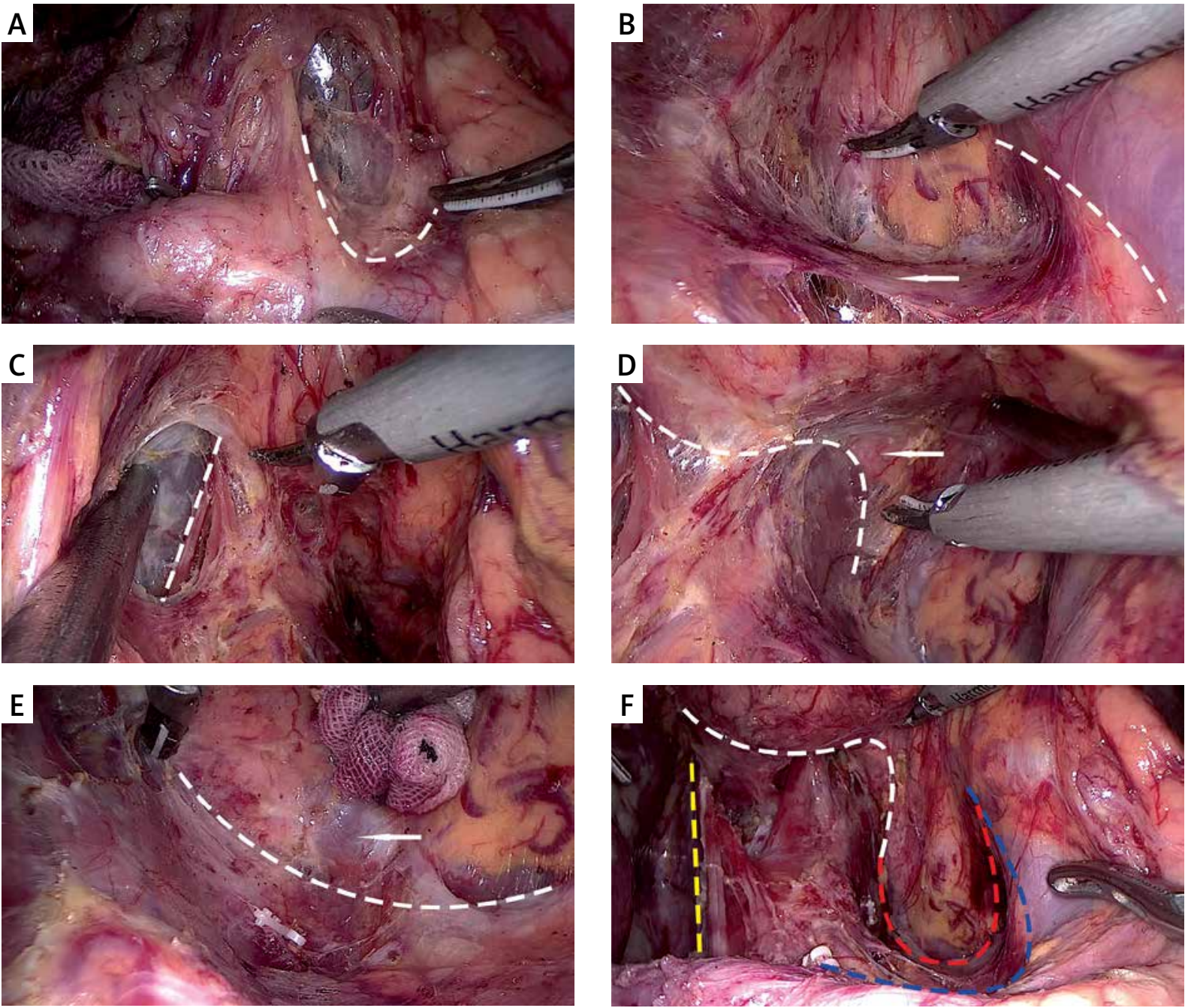

Photo 1. An intraoperative image showing the main procedure, pathway and overall view of the "enjoyable space". A - The dashed line shows the pathway of the entrance located between the origin of the left gastric artery (LGA) and the splenic artery. B - The dashed line shows the pathway of the lower border extending along the splenic artery. The arrow indicates the left Gerota's fascia. C - The pathway of the right border from the back of the LGA to the right side of the esophageal hiatus. D - The dashed line shows the pathway of the upper border, lining the esophageal hiatus, the surface of the left crus of the diaphragm and the gastrophrenic ligament (GPL). The arrow indicates the GPL. E - The dashed line shows the pathway of the left border, exposing the posterior edge of the middle-upper spleen. The arrow indicates the spleen. $\mathbf{F}$ - The dashed line shows the overall view of the pathways of the "enjoyable space". The yellow dashed line indicates the right border, the white dashed line indicates the upper border, the red dashed line indicates the left border, and the blue dashed line indicates the lower border

the posterior gastric artery (PGA) and the left gastroepiploic vessels (LGEVs) (LN no. 4sb) were divided from their origin (Photos 2 A, B). Subsequently, the posterior wall of the fundus and body of the stomach were pulled toward the upper right to fully reveal the splenic hilum and the gastrosplenic ligament. The fatty lymphatic tissue within the splenic hilum (LN no. 10) was pulled up and gradually denuded from the surface of the inferior splenic lobar vessels (ISLVs) to the superior splenic lobar vessels (SSLVs). In this procedure, 3-4 branches of the short gastric arteries (SGA), which derive from the SSLVS, were divided at their roots (Photo $2 \mathrm{C}$ ). At this point, the dissection of LNs no. 10 and no. 

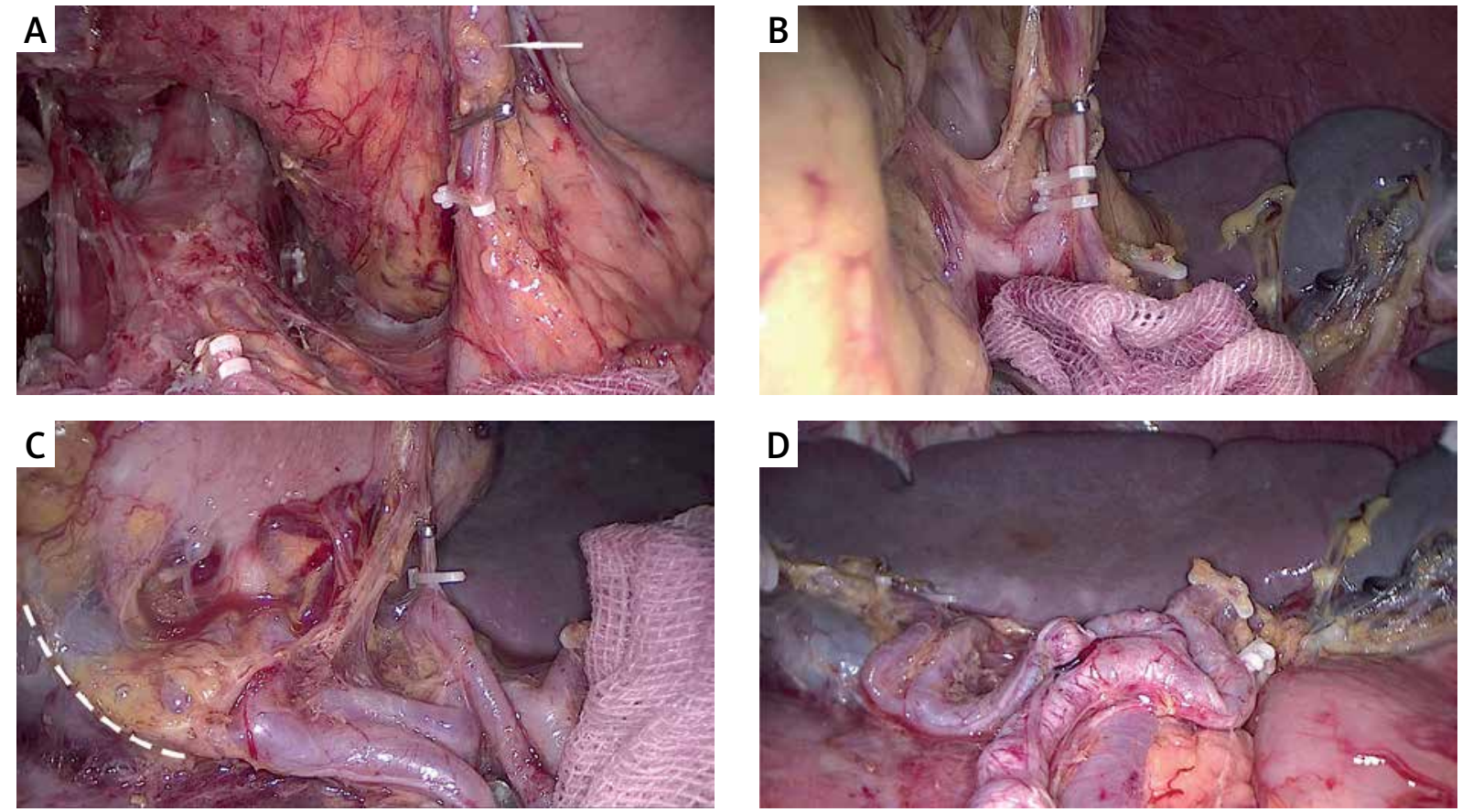

Photo 2. An intraoperative image showing the procedures used to dissect LNs no. 10 and no. 11 along the splenic vessels. A - Exposing and clipping the posterior gastric artery (PGA). The arrow indicates the dissected no. 11 LN. B - Exposing and clipping the left gastroepiploic vessels (LGEVs). C - Exposing and clipping the short gastric arteries (SGA). The dashed line shows the partial left border of the "enjoyable space". D - An overall view of the skeletonized splenic vessel trunk and branches

11 was completed (Photo 2 D). Then, The GPL was easily cut away.

5. The liver was held up to cut the hepatogastric ligament along the lower border of the liver and the LNs around the lesser curvature (no. 3) were removed. Finally, the phrenoesophageal membrane and both vagus nerves were divided to dissect LNs no. 1 and no. 2.

\section{Digestive tract reconstruction}

The duodenum was transected $2 \mathrm{~cm}$ below the pylorus with a 60-mm laparoscopic cartridge linear stapling device through the major hand port. A longitudinal laparotomy was performed using a 6-8 cm skin incision at the epigastrium, and the specimen was extracted from the peritoneal cavity. An omega-type esophagojejunostomy and two end-to-side jejunojejunostomies were performed using circular staplers.

\section{Statistical analysis}

All statistical analyses were performed using IBM SPSS Statistics version 20 (SPSS Inc., Chicago,
IL, USA). Data were reported as the mean \pm standard deviation (SD).

\section{Results}

\section{Patient characteristics}

The 79 patients included 63 (79.7\%) men and 16 (20.3\%) women with a mean age of $63.2 \pm 9.3$ years (range: 29-79 years) and a mean body mass index (BMI) of $22.31 \pm 3.35 \mathrm{~kg} / \mathrm{m}^{2}$ (range: $14.36-34.72 \mathrm{~kg} / \mathrm{m}^{2}$ ). The tumor locations included the cardia $(n=51)$, the upper stomach area $(n=25)$, and the fundus $(n=3)$. The mean tumor size was $3.50 \pm 1.98 \mathrm{~cm}$ (range: $0.3-11 \mathrm{~cm}$ ). The postoperative pathological TNM stages included pT1 $(n=29)$, pT2 $(n=19)$, pT3 $(n=12)$, and pT4a $(n=19) ;$ pNO $(n=57)$, pN1 $(n=9), p N 2(n=10)$, and pN3 $(n=3) ; \mathrm{IA}(n=27)$, IB $(n=14)$, IIA $(n=14)$, IIB $(n=12)$, IIIA $(n=5)$, IIIB $(n=4)$, and IIIC $(n=3)$ (Table I).

\section{Intraoperative and postoperative outcomes}

For all the patients, the mean operation time was $232.5 \pm 46.0 \mathrm{~min}$, and the mean estimated blood loss was $67.6 \pm 52.3 \mathrm{ml}$. In all cases, the upper incisal 
margin was negative, and the total number of LNs removed from 79 patients was 2245 , with a mean of $28.1 \pm 10.8$ LNs retrieved from each patient. The mean times to first flatus, fluid diet, and soft diet were $3.4 \pm 0.8$ days, $4.5 \pm 0.7$ days and $7.1 \pm 0.6$ days, respectively, and the mean postoperative hospital stay was $10.3 \pm 1.6$ days (Table II).

Table I. Demographic features and clinicopathological characteristics of LPMET with "enjoyable space" in gastric cancer

\begin{tabular}{|c|c|}
\hline Variable & Value \\
\hline \multicolumn{2}{|l|}{ Gender, $n(\%)$ : } \\
\hline Male & $63(79.7)$ \\
\hline Female & $16(20.3)$ \\
\hline Age [years] & $63.2 \pm 9.3$ \\
\hline $\mathrm{BMI}\left[\mathrm{kg} / \mathrm{m}^{2}\right]$ & $22.31 \pm 3.35$ \\
\hline Tumor size $[\mathrm{cm}]$ & $3.50 \pm 1.98$ \\
\hline \multicolumn{2}{|c|}{ Tumor location, $n(\%)$ : } \\
\hline Cardia: & $51(64.6)$ \\
\hline Fundus & $3(3.8)$ \\
\hline Body & $25(31.6)$ \\
\hline \multicolumn{2}{|c|}{ Tumor depth (pT): } \\
\hline pT1 & $29(36.7)$ \\
\hline pT2 & $19(24.1)$ \\
\hline pT3 & $12(15.1)$ \\
\hline pT4a & $19(24.1)$ \\
\hline \multicolumn{2}{|c|}{ Lymph node metastasis (pN): } \\
\hline pNO & $57(72.2)$ \\
\hline $\mathrm{pN1}$ & $9(11.4)$ \\
\hline $\mathrm{pN2}$ & $10(12.6)$ \\
\hline pN3 & $3(3.8)$ \\
\hline \multicolumn{2}{|l|}{ TNM stage: } \\
\hline$\overline{I A}$ & $27(34.2)$ \\
\hline$\overline{I B}$ & $14(17.7)$ \\
\hline$\| \mathrm{A}$ & $14(17.7)$ \\
\hline $\mathrm{IIB}$ & $12(15.2)$ \\
\hline$I I I A$ & $5(6.3)$ \\
\hline IIIB & $4(5.1)$ \\
\hline IIIC & $3(3.8)$ \\
\hline
\end{tabular}

LPMET - laparoscopic perigastric mesogastrium excision technique, BMI body mass index, TNM - tumor (topography), $N$-lymph node, $M$ - metastasis.

\section{Morbidity and mortality}

Fourteen patients experienced postoperative complications resulting in a morbidity rate of $17.7 \%$ (Table III). These complications, which comprised pulmonary infection in 10 patients, paroxysmal atrial fibrillation in 2 patients and postoperative delirium in 2 patients, were all successfully treated using conservative methods. None of the patients experienced spleen injury, anastomotic leakage, chylous fistula, or abdominal infection. The intraoperative and postoperative mortality rates for the total patient population were $0 \%$.

\section{Short-term data}

After a median follow-up period of 12 months (range: 7-18 months), 1 patient experienced liver

Table II. Intraoperative and postoperative outcomes of LPMET with "enjoyable space" in gastric cancer

\begin{tabular}{|lc|}
\hline Variable & Value \\
\hline Operation time [min] & $232.5 \pm 46.0$ \\
\hline Blood loss [ml] & $67.6 \pm 52.3$ \\
\hline Upper incisal margin, $n:$ & 0 \\
\hline Positive & 79 \\
\hline Negative & 2245 \\
\hline Total no. of retrieved LNs & $28.1 \pm 10.8$ \\
\hline Mean no. of retrieved LNs & $3.4 \pm 0.8$ \\
\hline Time to first flatus (d) & $4.5 \pm 0.7$ \\
\hline Time to fluid diet (d) & $7.1 \pm 0.6$ \\
\hline Time to soft diet (d) & $10.3 \pm 1.6$ \\
\hline Hospital stay $(d)$ & \\
\hline
\end{tabular}

LPMET - laparoscopic perigastric mesogastrium excision technique, LNs-lymph nodes.

Table III. Postoperative complications of LPMET with "enjoyable space" in gastric cancer

\begin{tabular}{|lcc|}
\hline Item & Value & Incidence (\%) \\
\hline Postoperative complications & 14 & 17.7 \\
\hline Pulmonary infection & 10 & 12.7 \\
\hline Postoperative delirium & 2 & 2.5 \\
\hline Paroxysmal atrial fibrillation & 2 & 2.5 \\
\hline
\end{tabular}

LPMET - laparoscopic perigastric mesogastrium excision technique. 
metastasis 6 months after surgery and is receiving chemotherapy; of the other 78 patients, none died or experienced tumor recurrence or metastasis.

\section{Discussion}

Due to the complexity of the perigastric mesogastrium and the narrow and deep space of the splenic hilum, CME of the perigastric mesogastrium and dissection of LNs no. 10 and no. 11 remain technically challenging aspects of LRTG. Although the surgical plane is important for surgeons, few studies have described and characterized the features of the perigastric mesogastrium $[8,10,11]$. According to special embryological characteristics, we identified the left Gerota's fascia as the surgical plane and established the "enjoyable space" by following its surface. After completely establishing this space, there were several notable advantages. First, anatomically, this space is a natural avascular zone containing loose connective tissue that favors tissue separation, rarely causes bleeding or peripheral organ injury during an operation and is conducive to keeping the visual field clear. Second, membrane anatomy is a basic requirement for $\mathrm{CME}$, and some surgeons have tried to achieve CME outside the bursa $[12,13]$, but unified technical standards were lacking. In our study, LPMET was performed on the specific surgical plane outside the posterior aspect of the lesser peritoneal sac and maximally resecting the perigastric mesogastrium that contains the supporting vascular and lymphatic systems of the posterior wall of the stomach. Therefore, LPMET might provide a new approach to CME access and would be beneficial for reducing the incidence of "metastasis V". Lastly, establishing the "enjoyable space" may facilitate the subsequent dissection of LNs no. 10 and no. 11. With improvements in the laparoscopic technique, some surgeons have performed laparoscopic spleen-preserving splenic hilar lymphadenectomy [6, 14]. However, in clinical practice, surgeons might lose their sense of direction and enter the wrong surgical plane, which would cause unexpected hemorrhage or peripheral organ injury, when dissecting LNs along only the splenic vessels' trunk and branches. Unlike conventional surgery, LPMET primarily establishes the "enjoyable space" and hollows out the perigastric space toward the posterior edge of the spleen. Moreover, the superior border of the splenic vessel trunk is exposed during the process of establishing the "enjoyable space". By isolating and pulling up the sur- rounding mesenteries and gastrophrenic ligament (GSL), the surgeon can comprehensively observe morphological characteristics and the variation of splenic vessels at different levels, which may be useful to completely skeletonize the splenic vessels and their branches for lymphadenectomy.

Technically, identifying the entrance and maintaining the integrity of the superior and posterior fascia are the primary difficulties facing LPMET, particularly for obese patients. In our study, we identified the origin of the LGA and splenic artery as a reference mark for the starting point for the approach to the entrance. The main reason is that these structures, which are easily found when pulling the LGA upward, are relatively fixed and rarely variant. Moreover, these structures and the anterior pancreas are covered by the same serosa that forms the posterior aspect of the lesser sac. Completely peeling this serosa is beneficial to reveal the entrance and maintain the integrity of the lesser sac. The left Gerota's fascia and the posterior aspect of the lesser sac are thin membranous structures and easily damaged. We have realized that steady and tacit teamwork plays an important role in keeping integrity of both fascias. First, due to this space being filled with loose connective tissues, proper tension would be useful to reveal the cutting edge. Therefore, the sustain point for exposure should be flexibly adjusted. Hence, we provided our recommended technique of pulling and exposure for assistants. Second, precisely separating and avoiding local in-depth the surgical plane are the guarantee of maintaining the continuity of the cutting edge and the integrity of both fascias. We suggest that the surgeon should meticulously separate the connective tissues along the surface of the left Gerota's fascia, using blunt and sharp dissection.

When evaluating the outcomes of LPMET, it is important to assess complications. Previous studies have reported that the overall postoperative complication rate of laparoscopic total gastrectomy was $6-36.7 \%$ [15-18]. In this study, we found that 14 of $79(17.7 \%)$ patients experienced postoperative complications, and no patient experienced surgery-related complications and died within 30 days of the follow-up, suggesting that LPMET was safe and did not increase intraoperative and postoperative morbidity and mortality rates.

Theoretically, this technique can also be applied to patients with prior abdominal surgery, with the 
exception of upper abdominal operations that have damaged this surgical plane. However, these data are unavailable because the patients enrolled in this study did not have prior abdominal surgery. Randomized controlled studies including larger patient groups with comparison to prior techniques are needed to confirm the advantages and long-term efficacy of LPMET.

\section{Conclusions}

This study suggested that LPMET and the "enjoyable space" could be a novel, minimally invasive approach and space to achieve a convenient CME access and provide benefit for the dissection of LNs no. 10 and no. 11 .

\section{Acknowledgments}

Chang-Yue Zheng and Zhi-Yong Dong contributed equally to this work.

The authors are thankful to Li-sang Fu and Rui Xu for collecting patients' follow-up data.

This study was sponsored by the Key Project of Science and Technology Plan (Natural Science Foundation) of Fujian Province, China (NO. 2018J01193) and the Campus Scientific Research Projects of Putian University (No. 2017041).

\section{Conflict of interest}

The authors declare no conflict of interest.

\section{References}

1. Lin JX, Lin JL, Zheng CH, et al. Short- and long-term outcomes of laparoscopy-assisted versus open total gastrectomy for gastric cancer: a propensity score-matched analysis. Oncotarget 2017; 8: 80029-38.

2. Hosogi H, Okabe H, Shinohara H, et al. Laparoscopic splenic hilar lymphadenectomy for advanced gastric cancer. Transl Gastroenterol Hepatol 2016; 1: 30.

3. Japanese Gastric Cancer Association. Japanese gastric cancer treatment guidelines 2014 (ver. 4). Gastric Cancer 2017; 20 1-19.

4. Son T, Kwon IG, Hyung WJ. Minimally invasive surgery for gastric cancer treatment: current status and future perspectives. Gut Liver 2014; 8: 229-36.

5. Huang CM, Chen QY, Lin JX, et al. Huang's three-step maneuver for laparoscopic spleen-preserving No. 10 lymph node dissection for advanced proximal gastric cancer. Chin I Cancer Res 2014; 26: 208-10.

6. Xie D, Liu L, Osaiweran H, et al. Detection and characterization of metastatic cancer cells in the mesogastrium of gastric cancer patients. PLoS One 2015; 10: e0142970.
7. Virgilio E, D’Antonio C, Balducci G. Mesogastrium recurrence as expression of the fifth metastatic route of gastric cancer. Med Hypotheses 2014; 82: 403-4.

8. Xie D, Gao C, Lu A, et al. Proximal segmentation of the dorsal mesogastrium reveals new anatomical implications for laparoscopic surgery. Sci Rep 2015; 5: 16287.

9. Washington $\mathrm{K}$. 7th edition of the AJCC cancer staging manual: stomach. Ann Surg Oncol 2010; 17: 3077-9.

10. Huang CM, Chen QY, Lin JX, et al. Laparoscopic spleen-preserving splenic hilar lymphadenectomy performed by following the perigastric fascias and the intrafascial space for advanced upper-third gastric cancer. PLoS One 2014; 9: e90345.

11. Moore KL, Persaud TVN, Torchia MG. The developing human: clinically oriented embryology. Saunders/Elsevier, Philadelphia 2008.

12. Xie D, Chaoran Yu C, Liu L, et al. Short-term outcomes of laparoscopic D2 lymphadenectomy with complete mesogastrium excision for advanced gastric cancer. Surg Endosc 2016; 30: 5138-9.

13. Zou L, Xiong W, Mo D, et al. Totally laparoscopic complete bursectomy and D2 lymphadenectomy in radical total gastrectomy: an outside bursa omentalis approach. Surg Endosc 2016; 30: 4152.

14. Son SY, Shin DJ, Park YS, et al. Spleen-preserving lymphadenectomy versus splenectomy in laparoscopic total gastrectomy for advanced gastric cancer. Surg Oncol 2017; 26: 207-11.

15. Kitagami H, Morimoto M, Nakamura K, et al. Technique of Roux-en-Y reconstruction using overlap method after laparoscopic total gastrectomy for gastric cancer: 100 consecutively successful cases. Surg Endosc 2016; 30: 4086-91.

16. Kim HB, Kim SM, Ha MH, et al. Comparison of reduced port totally laparoscopic-assisted total gastrectomy (Duet Tltg) and conventional laparoscopic-assisted total gastrectomy. Surg Laparosc Endosc Percutan Tech 2016; 26: e132-6.

17. Song JH, Choi YY, An JY, et al. Short-term outcomes of laparoscopic total gastrectomy performed by a single surgeon experienced in open gastrectomy: review of initial experience. J Gastric Cancer 2015; 15: 159-66.

18. Li P, Huang CM, Zheng $\mathrm{CH}$, et al. Laparoscopic spleen-preserving splenic hilar lymphadenectomy in 108 consecutive patients with upper gastric cancer. World J Gastroenterol 2014; 20 : 11376-83.

Received: 27.06.2018, accepted: 6.08.2018. 